\title{
Ensuring Our Future Doctors Are Resilient
}

The way in which healthcare is delivered in the UK is changing, as are the demographics of medical students and doctors in training, as highlighted in a recent GMC report. (1) Staff encounter increasing pressures in stretched care systems against a background of an ageing population with complex multi-morbidity and chronic conditions. Levels of poor mental health are generally on the rise, particularly among the young, and our social care systems are under significant strain. These factors, which are unlikely to change substantially in the foreseeable future, make for challenging work environments where doctoring can often be intensely stressful. This can undermine performance, and lead to absence, illness, and attrition of expensively trained professionals. It can potentially compromise optimal patient care.

Medical education should prepare the next generation of doctors for the realities of clinical practice. This includes encountering what Schön described as the "swampy lowlands where situations are confusing 'messes' incapable of technical solution." and uncertainties of clinical practice (2). Medical schools face significant challenges; notably that medical students have a higher prevalence of mental health issues than other student groups, and yet are less likely than their peers to seek help. Coupled with increasing scrutiny, accountability, and litigation against doctors, and an erosion of doctors' professional status and autonomy, this makes for an uncomfortable professional climate. In addition, doctors are expected to evaluate ever-accumulating research evidence and integrate novel management options into clinical practice, as well as undergo professional appraisal and revalidation of their professional competence.

Understandably, in a system where surveillance and measurement are now so core, students and their teachers place great emphasis on examination marks and decile rankings. Arguably this comes at the expense of "soft skills" and the unquantifiable "art of medicine," with the humanistic elements of medical education consequently under threat. Students are less likely to witness good examples of self-care by clinician role models in over-stretched work settings where presenteeism and "over-work" may have become the norm. Nor, for the sake of a sustainable working life, are they specifically learning to foster their own wellbeing. Unless there is investment both financially and culturally to help mitigate this, more students and doctors are likely to become exhausted and burn out.

We feel that medical schools have a duty of care to their students to minimise these risks and optimise the support available, thereby endeavouring to foster future practitioner wellbeing and resilience.

In many medical schools, students themselves are innovating and developing wellbeing initiatives. These include the use of social media as an accessible form of support and a basis for a supportive community; sharing experiences through downloadable podcasts; fostering better communication channels that promote transparency between the student body and teaching faculty. Suggestions for action and a "wellbeing curriculum" are broad-ranging and abundant. We suggest that teaching faculties should continue to develop forms of learning and support environments that are non-judgmental and provide "safe spaces" for critical reflection and discussion of challenges. 
Students and their teachers are aware of the "hidden curriculum-the processes, pressures and constraints which fall outside the formal curriculum, and which are often unarticulated or unexplored" (3) and are sceptical about the assumptions of doctors' invulnerability that colour medical education's emotional landscape. We suggest that medical educators should be authentic about the genuine difficulties they have faced and continue to face. Being exposed to examples of senior colleagues' humanity and fallibility may serve to reduce the distress and disappointment that can arise when students aim for what may be emotionally and professionally unattainable and unsustainable, particularly in a group comprising many perfectionists.

The journey from undergraduate to qualified trainee is lengthy and intense. The destination is a vocation with high emotional labour, where stress levels can be very high. If medical schools recognise this, then their duty of care necessitates that adequate support services be made available. In so doing, they should engage students in their own personal and professional development, and assure them that there is a transparent process for raising concerns and having them acted on. We believe that educating medical students and doctors to be resilient would be cost-effective; it has been estimated that the cost of training a person to foundation year level is a quarter of a million pounds and to consultant level twice that sum (4). The financial consequences of doctor impairment and absence or loss of costly professionals to the system could help attract the resources required for adapting curricula and improving student support systems.

Nationally and internationally, building medical student and doctor resilience will also deliver significant benefits to patients. Concerns must now extend out to the wider community of medical schools and other healthcare educators. In the interests of our profession's future and the sustainability of the NHS, the necessary sharing of ideas, best practice and wellbeing initiatives must transcend competition.

David Peters, Director, Westminster Centre for Resilience, University of Westminster

Christopher Horn, GP, Academic Clinical Fellow, Swansea University Medical School

Faye Gishen, Consultant Physician \& Academic Lead for Clinical \& Professional Practice, University College London Medical School

Competing interests: None declared

\section{$\underline{\text { References }}$}

(1) GMC (2018). https://www.gmc-uk.org/static/documents/content/SoMEP-2017-finalfull.pdf

(2) Schön D (1983). The Reflective Practitioner. Aldershot: Ashgate 
(3) Lempp H, Seale C (2004) The hidden curriculum in undergraduate medical education: qualitative study of medical students' perceptions of teaching. BMJ 2004;329:770

(4) https://www.bma.org.uk/news/media-centre/press-briefing-papers (2013 